\title{
Boundary regularity under generalized growth conditions
}

\author{
Petteri Harjulehto and Peter Hästö
}

\begin{abstract}
We study the Dirichlet $\varphi$-energy integral with Sobolev boundary values. The function $\varphi$ has generalized Orlicz growth. Special cases include variable exponent and double phase growths. We show that minimizers are regular at the boundary provided a weak capacity fatness condition is satisfied. This condition is satisfies for instance if the boundary is Lipschitz. The results are new even for Orlicz spaces.
\end{abstract}

Keywords. Dirichlet energy integral, regular boundary point, minimizer, superminimizer, generalized Orlicz space, Musielak-Orlicz spaces, the weak Harnack inequality, nonstandard growth, variable exponent, double phase

Mathematics Subject Classification (2010). 49N60, 35J66, 35J20

\section{Introduction}

We study the Dirichlet energy integral in a bounded domain $\Omega \subset \mathbb{R}^{n}$ with Sobolev boundary values:

$$
\inf \int_{\Omega} \varphi(x,|\nabla u|) d x
$$

where the infimum is taken over all $u \in W^{1, \varphi(\cdot)}(\Omega)$ with $u-f \in W_{0}^{1, \varphi(\cdot)}(\Omega)$. The function $\varphi$ has generalized Orlicz growth and satisfies conditions (A0), (A1), (A1-n), (aInc) and (aDec) that have been previously used in $[9,15,17,20]$. Our results include as special cases the constant exponent case $\varphi(x, t)=t^{p}$, the variable exponent case $\varphi(x, t)=t^{p(x)}$ and the double phase case $\varphi(x, t)=$ $t^{p}+a(x) t^{q}$. Such problems have been recently studied e.g. in $[1,3,5,7,8,12,14$, $21,25,26]$. For a detailed motivation of our context and additional references, we refer to the introduction of [18].

P. Harjulehto: Department of Mathematics and Statistics, FI-20014 University of Turku, Finland; petteri.harjulehto@utu.fi

P. Hästö: Department of Mathematics and Statistics, FI-20014 University of Turku, Finland and Department of Mathematics, FI-90014 University of Oulu, Finland; peter.hasto@oulu.fi (corresponding author) 
Our main result says that if the complement of $\Omega$ is locally fat at $x_{0} \in \partial \Omega$ in the capacity sense, then the boundary point is regular, i.e at this point the boundary value is attained not only in the Sobolev sense but point-wise. The main theorem yields for example that every boundary point is regular in Lipschitz domains and Hölder domains with appropriate exponent. To the best of our knowledge, the result is new even in the Orlicz case, $\varphi(x, t)=\varphi(t)$.

Theorem 1.1. Let $\Omega \subset \mathbb{R}^{n}$ be bounded and $x_{0} \in \partial \Omega$. Let $\varphi \in \Phi\left(\mathbb{R}^{n}\right)$ be strictly convex and satisfy (A0), (A1), (A1-n), (aInc) and (aDec). If there exists $c \in(0,1)$ and $R>0$ such that

$C_{\varphi(\cdot)}\left(B\left(x_{0}, r\right) \backslash \Omega, B\left(x_{0}, 2 r\right)\right) \geqslant c C_{\varphi(\cdot)}\left(B\left(x_{0}, r\right), B\left(x_{0}, 2 r\right)\right) \quad$ for all $\quad 0<r<R$,

then $x_{0}$ is a regular boundary point.

The proof of the main theorem is based on the properties of superminimizers of the Dirichlet $\varphi$-energy integral. Following the proofs of our previous paper [18], we show that superminimizers are locally bounded below, Corollary 3.4, and satisfy the weak Harnack inequality, Theorem 4.3. Using the supremumestimates and the weak Harnack inequality, we show that every superminimizer has a lower semicontinuous representative, and if additionally the superminimizer is bounded then for lower semicontinuous representative every point is a Lebesgue point, Theorem 4.4. Then we study continuity of superminimizers in Theorem 5.2 and show that for every $\varepsilon>0$

$$
\frac{C_{\varphi(\cdot)}\left(B\left(x_{0}, r\right) \cap\left\{\left|u-u\left(x_{0}\right)\right|>\varepsilon\right\}, B\left(x_{0}, 2 r\right)\right)}{C_{\varphi(\cdot)}\left(B\left(x_{0}, r\right), B\left(x_{0}, 2 r\right)\right)} \rightarrow 0
$$

as $r \rightarrow 0^{+}$. The lower semicontinuity and the above capacity density condition of continuity for superminimizers prove together with the pasting lemma the main theorem, cf. page 22.

As can be seen, the steps in our proof correspond to the constant exponent case. However, our minimizer is not homogeneous, so we cannot use techniques based on scaling. Therefore, we have combined arguments, mainly from [4,23], which are not crucially based on scaling, and in some cases modified them (e.g. the test function in the proof of Lemma 5.1).

\section{Preliminaries}

By $\Omega \subset \mathbb{R}^{n}$ we denote a bounded domain, i.e. an open and connected set. By $A \Subset \Omega$ we mean that $A$ is compactly contained in $\Omega$, i.e. there exists a compact set $K$ with $A \subset K \subset \Omega$. The notation $f \lesssim g$ means that there exists a constant $C>0$ such that $f \leqslant C g$. The notation $f \approx g$ means that $f \lesssim g \lesssim f$. By 
$c$ we denote a generic constant whose value may change between appearances. A function $f$ is almost increasing if there exists a constant $L \geqslant 1$ such that $f(s) \leqslant L f(t)$ for all $s \leqslant t$ (abbreviated $L$-almost increasing). Almost decreasing is defined similarly.

Generalized Orlicz spaces $L^{\varphi(\cdot)}$ have been studied since the 1940s. A major synthesis of functional analysis in these spaces is given in the 1983 monograph of Musielak [24], hence the alternative name Musielak-Orlicz spaces. Following ideas by Maeda, Mizuta, Ohno and Shimomura (e.g. [22]) we have studied these spaces from a point-of-view which emphasizes the possibility of choosing appropriately the $\Phi$-function generating the norm in the space. In this perspective, some classical concepts, like convexity, are too rigid. Hence we have arrived at the following definition.

Definition 2.1. We say that $\varphi: \Omega \times[0, \infty) \rightarrow[0, \infty]$ is a weak $\Phi$-function, and write $\varphi \in \Phi_{w}(\Omega)$, if the following conditions hold

- For every $t \in[0, \infty)$ the function $x \mapsto \varphi(x, t)$ is measurable and for every $x \in \Omega$ the function $t \mapsto \varphi(x, t)$ is non-decreasing and left-continuous.

- $\varphi(x, 0)=\lim _{t \rightarrow 0^{+}} \varphi(x, t)=0$ and $\lim _{t \rightarrow \infty} \varphi(x, t)=\infty$ for every $x \in \Omega$.

- The function $t \mapsto \frac{\varphi(x, t)}{t}$ is $L$-almost increasing for $t>0$ uniformly in $\Omega$. "Uniformly" means that $L$ is independent of $x$.

If $\varphi \in \Phi_{w}(\Omega)$ is additionally convex, then $\varphi$ is a $\Phi$-function, and we write $\varphi \in \Phi(\Omega)$.

Two functions $\varphi$ and $\psi$ are equivalent, $\varphi \simeq \psi$, if there exists $L \geqslant 1$ such that $\psi\left(x, \frac{t}{L}\right) \leqslant \varphi(x, t) \leqslant \psi(x, L t)$ for every $x \in \Omega$ and every $t>0$. Equivalent $\Phi$-functions give rise to the same space with comparable norms.

We say that $\varphi$ is doubling if there exists a constant $L \geqslant 1$ such that $\varphi(x, 2 t) \leqslant L \varphi(x, t)$ for every $x \in \Omega$ and every $t>0$. If $\varphi$ is doubling with constant $L$, then by iteration

$$
\varphi(x, t) \leqslant L^{2}\left(\frac{t}{s}\right)^{Q} \varphi(x, s)
$$

for every $x \in \Omega$ and every $0<s<t$, where $Q=\log _{2}(L)$, e.g. [4, Lemma 3.3]. If $\varphi$ is doubling, then $(1)$ yields that $\simeq$ implies $\approx$. On the other hand, $\approx$ always implies $\simeq$ since the function $t \mapsto \frac{\varphi(x, t)}{t}$ is almost increasing; hence $\simeq$ and $\approx$ are equivalent in the doubling case. Note that doubling also yields that $\varphi(x, t+s) \lesssim \varphi(x, t)+\varphi(x, s)$.

Assumptions. Let us write $\varphi_{B}^{+}(t):=\sup _{x \in B} \varphi(x, t)$ and $\varphi_{B}^{-}(t):=\inf _{x \in B} \varphi(x, t)$; and abbreviate $\varphi^{ \pm}:=\varphi_{\Omega}^{ \pm}$. We state some assumptions for later reference.

(A0) There exists $\beta \in(0,1)$ such that $\varphi^{+}(\beta) \leqslant 1 \leqslant \varphi^{-}(1)$. 
(A1) There exists $\beta \in(0,1)$ such that, for every ball $B \subset \Omega$,

$$
\varphi_{B}^{+}(\beta t) \leqslant \varphi_{B}^{-}(t) \quad \text { when } \quad t \in\left[1,\left(\varphi_{B}^{-}\right)^{-1}\left(\frac{1}{|B|}\right)\right] .
$$

(A1-n) There exists $\beta \in(0,1)$ such that, for every ball $B \subset \Omega$,

$$
\varphi_{B}^{+}(\beta t) \leqslant \varphi_{B}^{-}(t) \quad \text { when } \quad t \in\left[1, \frac{1}{\operatorname{diam} B}\right]
$$

(aInc) There exist $p>1$ and $L \geqslant 1$ such that $t \mapsto \frac{\varphi(x, t)}{t^{p}}$ is $L$-almost increasing in $(0, \infty)$.

(aDec) There exist $q>1$ and $L \geqslant 1$ such that $t \mapsto \frac{\varphi(x, t)}{t^{q}}$ is $L$-almost decreasing in $(0, \infty)$.

We write (Inc) if the ratio is increasing rather than just almost increasing, similarly for (Dec). All these assumptions are invariant under equivalence of $\Phi$-functions. Note that the optimal $p$ and $q$ correspond to the lower and upper Matuszewska-Orlicz indexes, respectively.

Furthermore, $(\mathrm{A} 0)$ and (aDec) imply that $\varphi(x, 1) \lesssim \beta^{-q} \varphi(x, \beta) \leqslant \beta^{-q}$, so this together with $1 \leqslant \varphi^{-}(1)$ yields that $\varphi(x, 1) \approx 1$. By Lemma 2.6 of [18] doubling is equivalent to (aDec). The conditions (A1) and (A1-n) can be used also in cubes instead of balls, see Lemmas 2.10 and 2.11 in [18].

Example 2.2. Let us consider the assumptions in some important special cases, namely variable exponent growth and double phase growth. The next table contains a interpretation of the assumptions for four $\Phi$-functions. Note that in many cases the condition in the special case is a nearly optimal sufficient condition: for instance, in the variable exponent case $p \in C^{\text {log }}$ implies (A1), and no worse continuity modulus is sufficient, but there may be exponents $p \notin C^{\log }$ for which (A1) nevertheless holds. [2, 6, 20, 27]

\begin{tabular}{l|ccccc}
$\varphi(x, t)$ & $(\mathrm{A} 0)$ & $(\mathrm{A} 1)$ & $(\mathrm{A} 1-n)$ & $(\mathrm{aInc})$ & $(\mathrm{aDec})$ \\
\hline$t^{p(x)} a(x)$ & $a \approx 1$ & $p \in C^{\log }$ & $p \in C^{\log }$ & $p^{-}>1$ & $p^{+}<\infty$ \\
$t^{p(x)} \log (e+t)$ & $\operatorname{true}$ & $p \in C^{\log }$ & $p \in C^{\log }$ & $p^{-}>1$ & $p^{+}<\infty$ \\
$t^{p}+a(x) t^{q}$ & $a \in L^{\infty}$ & $a \in C^{\frac{n}{p}(q-p)}$ & $a \in C^{q-p}$ & $p>1$ & $q<\infty$ \\
$t^{p}+a(x) t^{p} \log (e+t)$ & $a \in L^{\infty}$ & $a \in C^{\log }$ & $a \in C^{\log }$ & $p>1$ & $p<\infty$
\end{tabular}

Generalized Orlicz spaces. The generalized Orlicz and Orlicz-Sobolev spaces have been studied with our assumptions in $[9,15,17,20]$. We recall some definitions. We denote by $L^{0}(\Omega)$ the set of measurable functions in $\Omega$.

Definition 2.3. Let $\varphi \in \Phi_{w}(\Omega)$ and define the modular $\varrho_{\varphi(\cdot)}$ for $f \in L^{0}(\Omega)$ by

$$
\varrho_{\varphi(\cdot)}(f):=\int_{\Omega} \varphi(x,|f(x)|) d x .
$$


The generalized Orlicz space, also called Musielak-Orlicz space, is defined as the set

$$
L^{\varphi(\cdot)}(\Omega):=\left\{f \in L^{0}(\Omega): \lim _{\lambda \rightarrow 0^{+}} \varrho_{\varphi(\cdot)}(\lambda f)=0\right\}
$$

equipped with the (Luxemburg) norm

$$
\|f\|_{L^{\varphi(\cdot)}(\Omega)}:=\inf \left\{\lambda>0: \varrho_{\varphi(\cdot)}\left(\frac{f}{\lambda}\right) \leqslant 1\right\} .
$$

If the set is clear from the context we abbreviate $\|f\|_{L^{\varphi(\cdot)}(\Omega)}$ by $\|f\|_{\varphi(\cdot)}$.

Hölder's inequality holds in generalized Orlicz spaces with a constant 2, without restrictions on the $\Phi_{w}$-function [10, Lemma 2.6.5]:

$$
\int_{\Omega}|f||g| d x \leqslant 2\|f\|_{\varphi(\cdot)}\|g\|_{\varphi^{*}(\cdot)}
$$

Definition 2.4. A function $u \in L^{\varphi(\cdot)}(\Omega)$ belongs to the Orlicz-Sobolev space $W^{1, \varphi(\cdot)}(\Omega)$ if its weak partial derivatives $\partial_{1} u, \ldots, \partial_{n} u$ exist and belong to the space $L^{\varphi(\cdot)}(\Omega)$.

To study boundary value problems, we need a concept of weak boundary value spaces.

Definition 2.5. $W_{0}^{1, \varphi(\cdot)}(\Omega)$ is the closure of $C_{0}^{\infty}(\Omega)$ in $W^{1, \varphi(\cdot)}(\Omega)$.

If $\varphi \in \Phi_{w}$ satisfies (A0) and (aInc) and $\Omega \subset \mathbb{R}^{n}$ is bounded, then $L^{\varphi(\cdot)}(\Omega) \hookrightarrow$ $L^{p}(\Omega), W^{1, \varphi(\cdot)}(\Omega) \hookrightarrow W^{1, p}(\Omega)$ and $W_{0}^{1, \varphi(\cdot)}(\Omega) \hookrightarrow W_{0}^{1, p}(\Omega)[17$, Lemmas 4.4, 6.2 and 6.9].

We need the following fact regarding Sobolev functions. The assumptions are needed because smooth functions are not necessary dense in the OrliczSobolev space and in this case our definition for zero boundary values OrliczSobolev space is deficient.

Lemma 2.6 (Lemma 3.4, [18]). Let $\Omega \subset \mathbb{R}^{n}$. Let $\varphi \in \Phi_{w}(\Omega)$ satisfy (A0), (A1) and $(a D e c)$. If $v \in W^{1, \varphi(\cdot)}(\Omega)$ and $\operatorname{spt} v \subset \Omega$, then $v \in W_{0}^{1, \varphi(\cdot)}(\Omega)$.

Capacity and fine properties of functions. Fine properties of Sobolev functions can be studied by different capacities. Here we use a relative capacity defined as follows.

Definition 2.7. Let $E \Subset \Omega$. Then the relative Sobolev capacity of $E$ is defined by

$$
C_{\varphi(\cdot)}(E, \Omega):=\inf _{u \in S_{\varphi(\cdot)}(E, \Omega)} \int_{\Omega} \varphi(x,|\nabla u|) d x
$$

where the infimum is taken over the set $S_{\varphi(\cdot)}(E, \Omega)$ of all functions $u \in W_{0}^{1, \varphi(\cdot)}(\Omega)$ with $u \geqslant 1$ in an open set containing $E$. 
Standard arguments yield the following properties for the capacity (see, e.g., [10, Chapter 10] and [25]). Properties (C1)-(C5) need only the assumption $\varphi \in \Phi_{w}(\Omega)$, for (C6) and (C7) we need to assume that (aDec) and (aInc) hold (cf. [16]).

(C1) $C_{\varphi(\cdot)}(\emptyset, \Omega)=0$.

(C2) If $E_{1} \subset E_{2} \Subset \Omega$, then $C_{\varphi(\cdot)}\left(E_{1}, \Omega\right) \leqslant C_{\varphi(\cdot)}\left(E_{2}, \Omega\right)$.

(C3) If $E \Subset \Omega$, then

$$
C_{\varphi(\cdot)}(E, \Omega)=\inf _{\substack{E \subset U \\ U \text { open }}} C_{\varphi(\cdot)}(U, \Omega) .
$$

(C4) If $E_{1}, E_{2} \Subset \Omega$, then

$$
C_{\varphi(\cdot)}\left(E_{1} \cup E_{2}, \Omega\right)+C_{\varphi(\cdot)}\left(E_{1} \cap E_{2}, \Omega\right) \leqslant C_{\varphi(\cdot)}\left(E_{1}, \Omega\right)+C_{\varphi(\cdot)}\left(E_{2}, \Omega\right) .
$$

(C5) If $K_{1} \supset K_{2} \supset \cdots$ are compact sets in $\Omega$, then

$$
\lim _{i \rightarrow \infty} C_{\varphi(\cdot)}\left(K_{i}, \Omega\right)=C_{\varphi(\cdot)}\left(\cap_{i=1}^{\infty} K_{i}, \Omega\right)
$$

(C6) For $E_{1} \subset E_{2} \subset \ldots$ compactly contained in $\Omega$,

$$
\lim _{i \rightarrow \infty} C_{\varphi(\cdot)}\left(E_{i}, \Omega\right)=C_{\varphi(\cdot)}\left(\cup_{i=1}^{\infty} E_{i}, \Omega\right) .
$$

(C7) For $E_{i} \Subset \Omega$,

$$
C_{\varphi(\cdot)}\left(\cup_{i=1}^{\infty} E_{i}, \Omega\right) \leqslant \sum_{i=1}^{\infty} C_{\varphi(\cdot)}\left(E_{i}, \Omega\right) .
$$

We next estimate the capacity of a ball. Note that the upper and lower bounds are comparable under assumption (A1-n).

Lemma 2.8. Let $\varphi \in \Phi_{w}(2 B)$ be doubling. If $B$ is a ball with a radius $r$, then

$$
|B| \varphi_{2 B}^{-}\left(\frac{1}{r}\right) \lesssim C_{\varphi(\cdot)}(B, 2 B) \lesssim|B| \varphi_{2 B}^{+}\left(\frac{1}{r}\right) .
$$

Proof. Let $u \in W_{0}^{1, \varphi(\cdot)}(2 B)$ be such that $0 \leqslant u \leqslant 1, u=1$ in $B$ and $|\nabla u| \lesssim \frac{1}{r}$. Then by doubling we obtain

$$
C_{\varphi(\cdot)}(B, 2 B) \leqslant \int_{2 B} \varphi(x,|\nabla u|) d x \leqslant \int_{2 B} \varphi_{2 B}^{+}\left(\frac{c}{r}\right) d x \lesssim|B| \varphi_{2 B}^{+}\left(\frac{1}{r}\right) .
$$

For the opposite inequality, we obtain by Lemma 4.3 of [20] and the definition of 1-capacity that

$$
\begin{aligned}
\int_{2 B} \varphi(x,|\nabla u|) d x & \geqslant \int_{2 B} \varphi_{2 B}^{-}(|\nabla u|) d x=|2 B| f_{2 B} \varphi_{2 B}^{-}(|\nabla u|) d x \\
& \geqslant|2 B| \varphi_{2 B}^{-}\left(\frac{1}{2} f_{2 B}|\nabla u| d x\right) \geqslant|2 B| \varphi_{2 B}^{-}\left(\frac{C_{1}(B, 2 B)}{2|2 B|}\right) .
\end{aligned}
$$


Since $C_{1}(B, 2 B) \approx r^{n-1}$ (e.g., Theorem 4.15, p. 175, [11]), we obtain by doubling that

$$
\int_{2 B} \varphi(x,|\nabla u|) d x \geqslant|2 B| \varphi_{2 B}^{-}\left(\frac{c}{r}\right) \gtrsim|B| \varphi_{2 B}^{-}\left(\frac{1}{r}\right)
$$

This concludes the proof.

A function $f: \Omega \rightarrow[-\infty, \infty]$ is $\varphi(\cdot)$-quasicontinuous in $D \Subset \Omega$ if for every $\varepsilon>0$ there is a set $E$ such that $C_{\varphi(\cdot)}(E, \Omega)<\varepsilon$ and $\left.f\right|_{D \backslash E}$ is continuous. We say that a claim holds $\varphi(\cdot)$-quasieverywhere if it holds everywhere except in a set of $\varphi(\cdot)$-capacity zero.

Suppose that $u$ can be approximated by continuous functions in $W^{1, \varphi(\cdot)}(D)$ (cf. next lemma). Then a standard argument (e.g. [10, Theorem 11.1.3]) shows that every $u \in W^{1, \varphi(\cdot)}(\Omega)$ has a representative, which is quasicontinuous in every $D \Subset \Omega$, provided that $\varphi \in \Phi(\Omega)$ satisfies (aInc) and (aDec).

Lemma 2.9. Let $\varphi \in \Phi_{w}(\Omega)$ satisfy (A0), (A1) and (aDec). Let $D \Subset \Omega$ be open. Then for every $u \in W^{1, \varphi(\cdot)}(\Omega)$, there exists a sequence of function from $C^{\infty}(D) \cap W^{1, \varphi(\cdot)}(D)$ converging to $u$ in $W^{1, \varphi(\cdot)}(D)$.

Proof. Since $D \Subset \Omega$ is bounded, we may choose a bounded quasiconvex $\Omega^{\prime}$ such that $D \subset \Omega^{\prime} \subset \Omega$. By Lemma 5.1 and Theorem 6.6 of [17], there exists a sequence of function from $C^{\infty}\left(\Omega^{\prime}\right) \cap W^{1, \varphi(\cdot)}\left(\Omega^{\prime}\right)$ converging to $u$ in $W^{1, \varphi(\cdot)}\left(\Omega^{\prime}\right)$. Restricting the functions to $D$ gives the claim. This concludes the proof.

If $u \in W_{0}^{1, \varphi(\cdot)}(D)$ and $D \subset \Omega$, then the zero extension of $u$ belongs to $W^{1, \varphi(\cdot)}(\Omega)$ since $u$ can be approximated by $C_{0}^{\infty}(D)$-functions. The next lemma concerns the opposite implication.

Lemma 2.10. Let $\varphi \in \Phi_{w}(\Omega)$ satisfy (AO), (A1), (aInc) and (aDec) and let $D \Subset \Omega$ be open. If $u \in W^{1, \varphi(\cdot)}(\Omega)$ and $u=0$ in $\Omega \backslash D$, then $u \in W_{0}^{1, \varphi(\cdot)}(D)$. Moreover, if $u$ is non-negative, then there exist non-negative $u_{i} \in W_{0}^{1, \varphi(\cdot)}(D)$ with $\operatorname{spt} u_{i} \Subset D,\left\{u_{i} \neq 0\right\} \subset\{u \neq 0\}$ and $u_{i} \rightarrow u$ in $W^{1, \varphi(\cdot)}(D)$.

Proof. Let $\Omega^{\prime}$ be an open set satisfying $\bar{D} \subset \Omega^{\prime} \Subset \Omega$. Let $u^{*}$ be the quasicontinuous representative of $u$ in $\Omega^{\prime}$. Since $u=0$ everywhere in $\Omega^{\prime} \backslash D$, we obtain that $u^{*}$ is zero quasieverywhere in $\Omega^{\prime} \backslash D$. From now on we use this quasicontinuous representative and denote it by $u$.

We show that $u$ can be approximated by Sobolev functions with compact support in $D$. If we can construct such a sequence for $\max \{u, 0\}$, then we can do it for $\min \{u, 0\}$, as well. Combining these results proves the assertion for $u=\max \{u, 0\}+\min \{u, 0\}$. We therefore assume that $u$ is non-negative. A short calculation show that $\min \{u, k\} \rightarrow u$ in $W^{1, \varphi(\cdot)}(\Omega)$ as $k \rightarrow \infty$ and thus we may assume that $u$ is bounded. 
Let $\delta>0$ and let $U$ be an open set such that $u$ restricted to $\Omega^{\prime} \backslash U$ is continuous and $C_{\varphi(\cdot)}(U, \Omega)<\delta$. Let $E:=\left\{x \in \Omega^{\prime} \backslash D: u(x) \neq 0\right\}$. By assumption $C_{\varphi(\cdot)}(E, \Omega)=0$. Let $\omega_{\delta} \in S_{\varphi(\cdot)}(U \cup E)$ be such that $0 \leqslant \omega_{\delta} \leqslant 1$ and $\varrho_{1, \varphi(\cdot)}\left(w_{\delta}\right)<\delta$. Then $\omega_{\delta}=1$ in an open set $V$ containing $U \cup E$. For $0<\varepsilon<1$ define $u_{\varepsilon}(x):=\max \{u(x)-\varepsilon, 0\}$. Since the function $u$ is zero at $x \in \partial D \backslash V$ and $u$ restricted to $\Omega \backslash V$ is continuous, we find $r_{x}>0$ such that $u_{\varepsilon}$ vanishes in $B\left(x, r_{x}\right) \backslash V$. If $x \in \partial D \cap V$, then we choose $r_{x}$ such that $B\left(x, r_{x}\right) \subset V$. Thus the function $\left(1-\omega_{\delta}\right) u_{\varepsilon}$ vanishes in $B\left(x, r_{x}\right) \cup V$ for each $x \in \partial D$, which yields that it vanishes in a neighborhood of $\Omega^{\prime} \backslash D$. We have

$$
\left\|u-\left(1-\omega_{\delta}\right) u_{\varepsilon}\right\|_{1, \varphi(\cdot)} \leqslant\left\|u-u_{\varepsilon}\right\|_{1, \varphi(\cdot)}+\left\|\omega_{\delta} u_{\varepsilon}\right\|_{1, \varphi(\cdot)} .
$$

Since

$$
\left\|u-u_{\varepsilon}\right\|_{1, \varphi(\cdot)} \leqslant \varepsilon\left\|\chi_{\mathrm{spt} u}\right\|_{\varphi(\cdot)}+\left\|\chi_{\{0<u(x) \leqslant \varepsilon\}} \nabla u\right\|_{\varphi(\cdot)},
$$

we see that this term goes to zero with $\varepsilon$. Since $\varphi$ satisfies (aDec), we find that

$$
\begin{aligned}
\varrho_{1, \varphi(\cdot)}\left(\omega_{\delta} u\right) & \leqslant \varrho_{\varphi(\cdot)}\left(\omega_{\delta} u\right)+c \varrho_{\varphi(\cdot)}\left(\left|\nabla \omega_{\delta}\right| u\right)+c \varrho_{\varphi(\cdot)}\left(\omega_{\delta}|\nabla u|\right) \\
& \lesssim(\sup u+1)^{q} \varrho_{1, \varphi(\cdot)}\left(\omega_{\delta}\right)+\varrho_{\varphi(\cdot)}\left(\omega_{\delta}|\nabla u|\right) \\
& \leqslant \delta(\sup u+1)^{q}+\varrho_{\varphi(\cdot)}\left(\omega_{\delta}|\nabla u|\right)
\end{aligned}
$$

Since $\omega_{\delta} \rightarrow 0$ in $L^{\varphi(\cdot)}(\Omega)$, as $\delta \rightarrow 0$, we can choose a subsequence $\omega_{\delta}$ which tends to 0 point-wise almost everywhere. Then $\varrho_{\varphi(\cdot)}\left(\omega_{\delta}|\nabla u|\right) \rightarrow 0$ by the dominated convergence theorem with $\varphi(x,|\nabla u|)$ as a majorant. Therefore $\varrho_{1, \varphi(\cdot)}\left(\omega_{\delta} u\right) \rightarrow 0$ and so also $\left\|\omega_{\delta} u\right\|_{1, \varphi(\cdot)} \rightarrow 0$ as $\delta \rightarrow 0$. Thus we see that $\left(1-\omega_{\delta}\right) u_{\varepsilon} \rightarrow u$ as $\varepsilon, \delta \rightarrow 0$.

We have shown that $u$ can be can approximated by functions in $W^{1, \varphi(\cdot)}(D)$ with compact support in $D$. These functions are in $W_{0}^{1, \varphi(\cdot)}(D)$ by Lemma 2.6, and so the claim follows from the fact that $W_{0}^{1, \varphi(\cdot)}(D)$ is closed. This concludes the proof.

Lemma 2.11. Let $\Omega \subset \mathbb{R}^{n}$ be bounded. Let $\varphi \in \Phi_{w}(\Omega)$ satisfy (A0), (A1) and (aDec). If $v \in W^{1, \varphi(\cdot)}(\Omega)$ is non-negative and $u \in W_{0}^{1, \varphi(\cdot)}(\Omega)$, then $\min \{v, u\} \in$ $W_{0}^{1, \varphi(\cdot)}(\Omega)$.

Proof. Since $W_{0}^{1, \varphi(\cdot)}(\Omega)$ is a Banach space, by Lemma 2.6 we need only show that $\min \{v, u\}$ can be approximated by $W^{1, \varphi(\cdot)}(\Omega)$-functions with compact supports in $\Omega$.

Let $\left(w_{i}\right)$ be a sequence of $C_{0}^{\infty}(\Omega)$-functions converging to $u$ in $W^{1, \varphi(\cdot)}(\Omega)$ and point-wise. We show that $\left(\min \left\{v, w_{i}\right\}\right)$ converges to $\min \{v, u\}$ in $W^{1, \varphi(\cdot)}(\Omega)$, which gives the claim since $\operatorname{spt}\left(\min \left\{v, w_{i}\right\}\right) \subset \operatorname{spt}\left(w_{i}\right) \subset \Omega$. 
Let $A:=\{v<u\}$ and $A_{i}:=\left\{v<w_{i}\right\}$. Since $w_{i} \rightarrow u$ point-wise, $A_{i} \rightarrow A$. We obtain for the gradients by the doubling of $\varphi$ that

$$
\begin{aligned}
\int_{\Omega} \varphi & \left(x,\left|\nabla \min \{v, u\}-\nabla \min \left\{v, w_{i}\right\}\right|\right) d x \\
= & \int_{A \cap A_{i}} \varphi(x, 0) d x+\int_{\Omega \backslash\left(A \cup A_{i}\right)} \varphi\left(x,\left|\nabla u-\nabla w_{i}\right|\right) d x \\
& +\int_{A \backslash A_{i}} \varphi\left(x,\left|\nabla v-\nabla w_{i}\right|\right) d x+\int_{A_{i} \backslash A} \varphi(x,|\nabla u-\nabla v|) d x \\
\leqslant & \int_{\Omega} \varphi\left(x,\left|\nabla u-\nabla w_{i}\right|\right) d x+c \int_{A \backslash A_{i}} \varphi(x,|\nabla v-\nabla u|) d x \\
& +c \int_{A \backslash A_{i}} \varphi\left(x,\left|\nabla u-\nabla w_{i}\right|\right) d x+\int_{A_{i} \backslash A} \varphi(x,|\nabla u-\nabla v|) d x \rightarrow 0
\end{aligned}
$$

as $i \rightarrow \infty$, since $\left|\nabla w_{i}\right| \rightarrow|\nabla u|$ in $L^{\varphi(\cdot)}(\Omega)$ and $A_{i} \rightarrow A$. The calculation for the functions is the same. This concludes the proof.

\section{Local boundedness}

Definition 3.1. Let $\varphi \in \Phi_{w}(\Omega)$. A function $u \in W_{\text {loc }}^{1, \varphi(\cdot)}(\Omega)$ is a local quasiminimizer of the $\varphi(\cdot)$-energy in $\Omega$ if there exists a constant $K \geqslant 1$ such that

$$
\int_{\{v \neq 0\}} \varphi(x,|\nabla u|) d x \leqslant K \int_{\{v \neq 0\}} \varphi(x,|\nabla(u+v)|) d x
$$

for all $v \in W^{1, \varphi(\cdot)}(\Omega)$ with $\operatorname{spt} v:=\overline{\{v \neq 0\}} \subset \Omega$.

If the inequality is assumed only for all non-negative or non-positive $v$, then $u$ is called a local quasisuperminimizer or local quasisubminimizer, respectively.

In this section we show that quasisubminimizers are locally bounded from above and quasisuperminimizers are locally bounded from below. Our arguments follow Section 4 of [18]. We use the following setup for the rest of this section. Suppose that $0 \in \Omega \subset \mathbb{R}^{n}$ and $0<R<R_{0} \leqslant \frac{1}{2}$. We write $Q_{R}:=Q(0, R)$ for the cube centered at 0 with side-length $2 R$,

$$
A_{R}:=A(k, R):=Q_{R} \cap\{u>k\} \quad \text { and } \quad u_{+}:=\max \{u, 0\} .
$$

Once we have our results for cubes centered at 0 , we can get the general result by translation. Note that the $\Phi$-function also has to be translated, since our space is not translation-invariant as such.

The following result was established for quasiminimizers in [18]. In fact, the proof presented in the reference needs only that $u$ be a quasisubminimizer. For completeness, the proof is included here. 
Lemma 3.2 (Caccioppoli inequality). Let $\varphi \in \Phi_{w}(\Omega)$ be doubling. Let $u$ be a local quasisubminimizer in $\Omega$. Then for all $k \in \mathbb{R}$ we have

$$
\int_{A(k, r)} \varphi\left(x,\left|\nabla(u-k)_{+}\right|\right) d x \leqslant C \int_{A(k, R)} \varphi\left(x, \frac{u-k}{R-r}\right) d x
$$

where $C>0$ depends only on the doubling constant of $\varphi$ and the quasiminimizing constant of $u$.

Proof. Let $r \leqslant t<s \leqslant R$ and $k \in \mathbb{R}$. Let $\eta \in C_{0}^{\infty}\left(Q_{s}\right)$ be such that $0 \leqslant \eta \leqslant 1$, $\eta=1$ in $Q_{t}$, and $|\nabla \eta| \leqslant \frac{2}{s-t}$. Denote $w:=(u-k)_{+}$and $v:=u-\eta w$. Note that $v \leqslant u$, and $v=u$ in $Q_{s} \backslash A_{s}$. Since $u$ is a local quasisubminimizer with constant $K$ and $-\eta w \leqslant 0$,

$$
\int_{A_{s}} \varphi(x,|\nabla u|) d x \leqslant K \int_{A_{s}} \varphi(x,|\nabla v|) d x .
$$

In $A_{s}, w=u-k$ so that $v=u(1-\eta)+\eta k$, and hence $\nabla v=(1-\eta) \nabla u-(u-k) \nabla \eta$. From this follows that in $A_{s}$ we have

$$
|\nabla v| \leqslant(1-\eta)|\nabla u|+|\nabla \eta|(u-k)_{+} \leqslant 2 \max \left\{(1-\eta)|\nabla u|,|\nabla \eta|(u-k)_{+}\right\} .
$$

By doubling (with constant $L$ ) and $|\nabla \eta| \leqslant \frac{2}{s-t}$, we get that

$$
\begin{aligned}
\varphi(x,|\nabla v|) & \leqslant \varphi(x, 2(1-\eta)|\nabla u|)+\varphi\left(x, 4 \frac{(u-k)_{+}}{s-t}\right) \\
& \leqslant L \varphi(x,(1-\eta)|\nabla u|)+L^{2} \varphi\left(x, \frac{(u-k)_{+}}{s-t}\right) .
\end{aligned}
$$

Combining the above inequalities, we find that

$$
\int_{A_{s}} \varphi(x,|\nabla u|) d x \leqslant L K \int_{A_{s}} \varphi(x,(1-\eta)|\nabla u|) d x+L^{2} K \int_{A_{s}} \varphi\left(x, \frac{(u-k)_{+}}{s-t}\right) d x .
$$

Since $t<s<R$, it follows that $A_{t} \subset A_{s} \subset A_{R}$, and so we obtain

$$
\int_{A_{t}} \varphi(x,|\nabla u|) d x \leqslant L K \int_{A_{s}} \varphi(x,(1-\eta)|\nabla u|) d x+L^{2} K \int_{A_{R}} \varphi\left(x, \frac{(u-k)_{+}}{s-t}\right) d x .
$$

On the right-hand side, we have $\varphi(x,(1-\eta)|\nabla u|)=\varphi(x, 0)=0$ in $Q_{t}$, and so

$$
\int_{A_{s}} \varphi(x,(1-\eta)|\nabla u|) d x=\int_{A_{s} \backslash A_{t}} \varphi(x,(1-\eta)|\nabla u|) d x \leqslant \int_{A_{s} \backslash A_{t}} \varphi(x,|\nabla u|) d x .
$$

Now we can use the hole-filling trick by adding $L K \int_{A_{t}} \varphi(x,|\nabla u|) d x$ to both sides of (3), ending with $L K+1$ of the integral on the left-hand side, and $L K$ on the right. After we divide with $L K+1$, we have

$$
\int_{A_{t}} \varphi(x,|\nabla u|) d x \leqslant \frac{L K}{L K+1} \int_{A_{s}} \varphi(x,|\nabla u|) d x+\frac{L^{2} K}{L K+1} \int_{A_{R}} \varphi\left(x, \frac{(u-k)_{+}}{s-t}\right) d x .
$$

The multiplier $\frac{L K}{L K+1}<1$, so the claim follows from telescoping lemma (cf. Lemma 4.2, [18]) as usual. This concludes the proof. 
Proposition 3.3 (Lemma 4.11, [18]). Let $\varphi \in \Phi_{w}(\Omega)$ satisfy (A0), (A1), (aInc) and (aDec). Suppose that $u \in W_{\mathrm{loc}}^{1, \varphi(\cdot)}(\Omega)$ satisfies the Caccioppoli inequality (2). Then there exists $R_{0} \in(0,1)$ such that

$$
\underset{Q_{R / 2}}{\operatorname{ess} \sup } u \leqslant k_{0}+1+c R^{-\frac{q}{\alpha p}}\left(\int_{Q_{R}} \varphi\left(x,\left(u-k_{0}\right)_{+}\right) d x\right)^{\frac{1}{p}}
$$

for every $k_{0} \in \mathbb{R}$ when $R \in\left(0, R_{0}\right]$. Here $R_{0}$ is such that $R_{0} \leqslant c(n)$ and $\varrho_{L^{\varphi(\cdot)}\left(Q_{3 R_{0}}\right)}(\nabla u) \leqslant 1$, and the constant $c$ depends only on the parameters in assumptions and the dimension $n$.

Lemma 3.2 and Proposition 3.3 yield that quasisubminimizers are locally bounded above. If $u$ is quasisuperminimizer then $-u$ is a quasisubminimizer. We obtain the following corollary.

Corollary 3.4. Let $\varphi \in \Phi_{w}(\Omega)$ satisfy (A0), (A1), (aInc) and (aDec). Then

1. quasisubminimizers are locally bounded from above, and

2. quasisuperminimizers are locally bounded from below.

The dependence on $R$ in Proposition 3.3 is not good. It is possible to rectify this situation and fix the homogeneity of the right hand side by a scaling argument, cf. Theorem 5.7 in [18]. With exactly the same arguments, we obtain the following results, previously proved for quasiminimizers, also for quasisubminimizers.

Theorem 3.5. Let $\varphi \in \Phi_{w}(\Omega)$ satisfy (A0), (A1-n) and (aDec). Suppose that $u \in W_{\mathrm{loc}}^{1, \varphi(\cdot)}(\Omega)$ is a local quasisubminimizer which is locally bounded from above. Then

$$
\underset{Q_{R / 2}}{\operatorname{ess} \sup } u-k \lesssim\left(f_{Q_{R}}(u-k)_{+}^{q} d x\right)^{\frac{1}{q}}+R
$$

when $R \in\left(0, R_{0}\right]$ and $k \in \mathbb{R}$. The implicit constant depends only on the parameters in assumptions, $n, R_{0}$ and $\operatorname{ess}_{\sup _{Q_{r}}} u$.

By standard arguments, the previous inequality can be "upgraded" to include any exponent on the right-hand side (cf. [18, Corollary 5.9]).

Corollary 3.6. Let $\varphi \in \Phi_{w}(\Omega), u \in W_{\mathrm{loc}}^{1, \varphi(\cdot)}(\Omega)$ and $R_{0}>0$ be as in Theorem 3.5. Then

$$
\underset{Q_{R / 2}}{\operatorname{ess} \sup } u-k \lesssim\left(f_{Q_{R}}(u-k)_{+}^{q} d x\right)^{\frac{1}{q}}+R,
$$

for every $R \in\left(0, R_{0}\right], k \in \mathbb{R}$ and $q \in(0, \infty)$. The implicit constant is independent of $R$ and depends on $q$ and on the parameters listed in Theorem 3.5.

Note that these results do not require the assumptions (A1) and (aInc), but instead rely on $u$ being locally bounded. The latter can be concluded from the former by Corollary 3.4. 


\section{Lower semincontinuity of quasisuperminimizers}

We denote

$$
D(k, r):=\left\{x \in Q\left(x_{0}, r\right): u(x)<k\right\},
$$

and start with some auxiliary estimates which were done in [18] for quasiminimizers. Again, the same proofs work, so we give only the first step, and refer the reader to the reference for the others.

Lemma 4.1. Let $\varphi \in \Phi_{w}(\Omega)$ satisfy (A0), (A1-n) and (aDec). Let $u \in$ $W_{\mathrm{loc}}^{1, \varphi(\cdot)}(\Omega)$ be a non-negative local quasisuperminimizer. Then there exist constants $\gamma_{0} \in(0,1)$ and $c>1$, depending only on the parameters in the assumptions, $n$ and $R_{0}$, such that if

$$
|D(\theta, R)| \leqslant \gamma_{0}\left|Q_{R}\right|
$$

for some $\theta>0$, then

$$
\underset{Q_{R / 2}}{\operatorname{essinf}} u+c R \geqslant \frac{\theta}{2}
$$

Proof. We observe that $-u$ is a quasisubminimizer bounded from above by 0 . Corollary 3.6 applied to $-u$, with $k=-\theta$ and $q=1$, implies that

$$
\underset{Q_{R / 2}}{\operatorname{ess} \sup }(-u)+\theta \leqslant C f_{Q_{R}}(\theta-u)_{+} d x+C R .
$$

Let $\gamma_{0}:=(2 C)^{-1}$. Then

$$
\begin{aligned}
\underset{Q_{R / 2}}{\operatorname{essinf} u+C R} & \geqslant \theta-\frac{C}{\left|Q_{R}\right|} \int_{D(\theta, R)}(\theta-u)_{+} d x \\
& \geqslant \theta-C \theta \frac{|D(\theta, R)|}{\left|Q_{R}\right|} \geqslant \theta-C \theta \gamma_{0}=\frac{\theta}{2} .
\end{aligned}
$$

This concludes the proof.

The following lemma is an improvement of the preceding one and the proof is the same as that of Lemma 6.2 in [18].

Lemma 4.2. Let $\varphi, u$ and $R_{0}$ be as in the previous lemma. Then for every $\kappa \in(0,1)$ there exists $\mu>0$ such that

$$
\left|D_{\theta}\right| \leqslant \kappa\left|Q_{R}\right| \quad \Rightarrow \quad \underset{Q_{R / 2}}{\operatorname{essinf}} u+c R \geqslant \mu \theta
$$

for all $R \in\left(0, R_{0}\right]$ and all $\theta>0$. 
Once we have the implication from Lemma 4.2, standard arguments yields the the following theorem, see for example Lemma 6.3 of [18] or Theorem 5.7 of [19] or pp. 239-240 in [13].

Theorem 4.3 (The weak Harnack inequality). Let $\varphi \in \Phi_{w}(\Omega)$ satisfy (A0), (A1-n), (aInc) and (aDec). Let $u \in W_{\mathrm{loc}}^{1, \varphi(\cdot)}(\Omega)$ be a nonnegative quasisuperminimizer in $\Omega$. Then there exists an exponent $h>0$ such that

$$
\left(f_{Q\left(x_{0}, R\right)} u^{h} d x\right)^{1 / h} \lesssim \operatorname{essinf}_{Q\left(x_{0}, R / 2\right)} u+R
$$

for every $R \leqslant c(n)$ with $Q\left(x_{0}, 3 R\right) \Subset \Omega$ and $\int_{Q\left(x_{0}, 3 R\right)} \varphi(x,|\nabla u|) d x \leqslant 1$. The implicit constant depends only on the parameters in the assumptions and $n$.

As an application of the weak Harnack inequality, we get the following result on lower semicontinuous representatives.

Theorem 4.4. Let $\varphi \in \Phi_{w}(\Omega)$ satisfy (A0), (A1-n), (aInc) and (aDec). Let $u$ be a local quasisuperminimizer which is bounded from below and set

$$
u^{*}(x):=\text { ess } \lim _{y \rightarrow x} \inf u(y) .
$$

Then $u^{*}$ is lower semicontinuous and $u=u^{*}$ almost everywhere.

If $u$ is additionally locally bounded, then every point is a Lebesgue point of $u^{*}$.

Proof. Standard arguments show that for any $u$, the function $u^{*}$ is lower semicontinuous, see for example p. 207 in [4].

Since $u \in W_{\text {loc }}^{1, \varphi(\cdot)}(\Omega) \subset L_{\text {loc }}^{1}(\Omega)$ we obtain by the Lebesgue differentiation theorem that the set

$$
E:=\left\{x_{0} \in \Omega:\left|u\left(x_{0}\right)\right|<\infty \text { and } \lim _{r \rightarrow 0} f_{Q\left(x_{0}, r\right)}\left|u(y)-u\left(x_{0}\right)\right| d y=0\right\}
$$

differs from $\Omega$ only by a set of Lebesgue measure zero. Since $x_{0} \in E$ is a Lebesgue point, we obtain that

$$
u^{*}\left(x_{0}\right)=\text { ess } \lim _{y \rightarrow x_{0}} \inf u(y) \leqslant \lim _{r \rightarrow 0} f_{Q\left(x_{0}, r\right)}|u(y)| d y=u\left(x_{0}\right) .
$$

We complete the proof of $u=u^{*}$ a.e. by showing that $u\left(x_{0}\right) \leqslant u^{*}\left(x_{0}\right)$ for all $x_{0} \in E$.

Note that $-u$ is a quasisubminimizer bounded from above. Thus Corollary 3.6 with $k=-u\left(x_{0}\right)$ yields

$$
\operatorname{esssup}_{Q\left(x_{0}, r / 2\right)}\left(u\left(x_{0}\right)-u\right) \lesssim f_{Q\left(x_{0}, r\right)}\left(u\left(x_{0}\right)-u\right)_{+} d x+r \leqslant f_{Q\left(x_{0}, r\right)}\left|u\left(x_{0}\right)-u\right| d x+r,
$$


provided $r$ is small enough. Therefore,

$$
u\left(x_{0}\right)-\underset{Q\left(x_{0}, r / 2\right)}{\operatorname{essinf}} u=\operatorname{essipp}_{Q\left(x_{0}, r / 2\right)}\left(u\left(x_{0}\right)-u\right) \lesssim f_{Q\left(x_{0}, r\right)}\left|u\left(x_{0}\right)-u\right| d x+r .
$$

Since $x_{0}$ is a Lebesgue point, the right hand side tends to zero as $r \rightarrow 0^{+}$. As above, we see that $\lim _{r \rightarrow 0^{+}} \operatorname{essinf}_{Q\left(x_{0}, r / 2\right)} u \leqslant u^{*}\left(x_{0}\right)$. Together, these gieve that $u\left(x_{0}\right) \leqslant u^{*}\left(x_{0}\right)$ and so $u=u^{*}$ a.e.

For the Lebesgue point property let $x_{0} \in \Omega$. Since $u^{*} \in W_{\text {loc }}^{1, \varphi(\cdot)}(\Omega)$ we may choose $R_{1}$ so small that

$$
\int_{Q\left(x_{0}, 3 R_{1}\right)} \varphi\left(x,\left|\nabla u^{*}\right|\right) d x \leqslant 1
$$

Since $u^{*}$ is lower semicontinuous and locally bounded, for every $\varepsilon>0$ there exist $R_{2}$ and $m>\varepsilon$ such that $u^{*}\left(x_{0}\right)-\varepsilon<u^{*}<m$ in $B\left(x_{0}, R_{2}\right)$. Let $R_{3}$ be so small that $m\left|B\left(x_{0}, R_{3}\right)\right| \leqslant 1$. Denote $v:=u^{*}-u^{*}\left(x_{0}\right)+\varepsilon$. Then $v$ is a quasisuperminimizer, $\nabla v=\nabla u^{*}$ and $0<v<2 m$. By Hölder's inequality we may assume that the exponent $h$ in the weak Harnack inequality is less than one. Thus the weak Harnack inequality (Theorem 4.3) yields for $R<$ $\min \left\{R_{1}, R_{2}, R_{3}\right\}$ that

$$
\begin{aligned}
f_{Q\left(x_{0}, R\right)}\left|u^{*}(x)-u^{*}\left(x_{0}\right)\right| d x \\
\quad=f_{Q\left(x_{0}, R\right)}\left|v(x)-v\left(x_{0}\right)\right| d x \leqslant v\left(x_{0}\right)+f_{Q\left(x_{0}, R\right)}|v| d x \\
=\varepsilon+f_{Q\left(x_{0}, R\right)}|v|^{1-h}|v|^{h} d x \leqslant \varepsilon+(2 m)^{1-h} f_{Q\left(x_{0}, R\right)}|v|^{h} d x \\
\quad \leqslant \varepsilon+C m^{1-h} \underset{Q\left(x_{0}, R / 2\right)}{\operatorname{essinf}} v^{h}+C m^{1-h} R \\
\leqslant \varepsilon+C m^{1-h} \varepsilon^{h}+C m^{1-h} R .
\end{aligned}
$$

Letting $R \rightarrow 0^{+}$and $\varepsilon \rightarrow 0^{+}$, we obtain that $x_{0}$ is a Lebesgue point of $u^{*}$. This concludes the proof.

The following lemma extends the class of permissible test functions.

Lemma 4.5. Let $\varphi \in \Phi_{w}(\Omega)$ satisfy (aDec) and let $u$ be a local quasisuperminimizer. Then

$$
\int_{\{v \neq 0\}} \varphi(x,|\nabla u|) d x \leqslant 2^{q} L K \int_{\{v \neq 0\}} \varphi(x,|\nabla(u+v)|) d x
$$

for all $v \in W^{1, \varphi(\cdot)}(\Omega)$ which can be approximated by a sequence of non-negative $v_{i} \in W^{1, \varphi(\cdot)}(\Omega)$ with $\operatorname{spt} v_{i} \Subset \Omega,\left\{v_{i} \neq 0\right\} \subset\{v \neq 0\}$ and $v_{i} \rightarrow v$ in $W^{1, \varphi(\cdot)}(\Omega)$ 
Proof. We may assume the the right-hand side is finite since otherwise there is nothing to prove. Let $v$ and $v_{i}$ be as in the statement of the result. We use $v_{i}$ as a test function:

$$
\int_{\left\{v_{i} \neq 0\right\}} \varphi(x,|\nabla u|) d x \leqslant K \int_{\left\{v_{i} \neq 0\right\}} \varphi\left(x,\left|\nabla\left(u+v_{i}\right)\right|\right) d x .
$$

On the other hand, we have the trivial inequality

$$
\int_{\{v \neq 0\} \backslash\left\{v_{i} \neq 0\right\}} \varphi(x,|\nabla u|) d x \leqslant K \int_{\{v \neq 0\} \backslash\left\{v_{i} \neq 0\right\}} \varphi\left(x,\left|\nabla\left(u+v_{i}\right)\right|\right) d x .
$$

since $\nabla v_{i}=0$ almost everywhere in $\{v \neq 0\} \backslash\left\{v_{i} \neq 0\right\} \subset\left\{v_{i}=0\right\}$. Since $\left\{v_{i} \neq 0\right\} \subset\{v \neq 0\}$, we obtain

$$
\begin{aligned}
\int_{\{v \neq 0\}} \varphi(x,|\nabla u|) d x & \leqslant \int_{\left\{v_{i} \neq 0\right\}} \varphi(x,|\nabla u|) d x+\int_{\{v \neq 0\} \backslash\left\{v_{i} \neq 0\right\}} \varphi(x,|\nabla u|) d x \\
& \leqslant K \int_{\{v \neq 0\}} \varphi\left(x,\left|\nabla\left(u+v_{i}\right)\right|\right) d x \\
& \leqslant 2^{q} L K \int_{\{v \neq 0\}} \varphi(x,|\nabla(u+v)|)+\varphi\left(x,\left|\nabla\left(v_{i}-v\right)\right|\right) d x
\end{aligned}
$$

by adding the two previous inequalities and by using (aDec). The claim follows from this as $i \rightarrow \infty$ since the second term goes to zero due to $\left\|v_{i}-v\right\|_{\varphi(\cdot)} \rightarrow 0$. This concludes the proof.

Even if one is interested in minimizers, sub- and superminimizers are often useful tools owing to their greater flexibility. One example of this is the following pasting result, which allows us to splice together two superminimizers. In the special case $D=\Omega$, the lemma yields that minimum of two quasisuperminimizers is a quasisuperminimizer. Naturally this yields the corresponding result for the maximum of two quasisubminimizers. The proof of the next lemma is based on Lemma 7.13 of [4].

Lemma 4.6 (Pasting lemma for quasisuperminimizers). Let $\varphi \in \Phi_{w}(\Omega)$ satisfy (A0), (A1), (aInc) and (aDec). Assume that $D \subset \Omega$ and that $u_{1}$ and $u_{2}$ are $K$-quasisuperminimizers in $D$ and $\Omega$, respectively. Let

$$
u:= \begin{cases}u_{2} & \text { in } \Omega \backslash D \\ \min \left\{u_{1}, u_{2}\right\} & \text { in } D .\end{cases}
$$

If $u \in W_{\mathrm{loc}}^{1, \varphi(\cdot)}(\Omega)$, then $u$ is a $2^{q} L K^{2}$-quasisuperminimizer. 
Proof. Let $\xi \in W^{1, \varphi(\cdot)}(\Omega)$ be non-negative test function with spt $\xi \subset \Omega$. Let $G:=\{\xi>0\}$ and $v:=u+\xi$. The claim is then that

$$
\int_{G} \varphi(x,|\nabla u|) d x \leqslant K^{2} \int_{G} \varphi(x,|\nabla v|) d x .
$$

Let $\Omega^{\prime} \Subset \Omega$ be an open set containing $\bar{G}$. Let $A:=\left\{u_{2}<v\right\}$ and note that $\left(v-u_{2}\right)_{+}=0$ in $\Omega \backslash A$. Since $u_{2}$ is a quasisuperminimizer in $\Omega$ and $A \subset G \Subset \Omega$ we obtain that

$$
\int_{A} \varphi\left(x,\left|\nabla u_{2}\right|\right) d x \leqslant K \int_{A} \varphi\left(x, \mid \nabla\left(u_{2}+\left(v-u_{2}\right)_{+} \mid\right) d x=K \int_{A} \varphi(x,|\nabla v|) d x .\right.
$$

Let $w:=\min \left\{u_{2}, v\right\}$ and $E:=\{w>u\}$. We observe that $w>u$ can only happen when $u<u_{2}$ and $\xi>0$, so we derive $E=\left\{x \in G \cap D: u_{1}(x)<u_{2}(x)\right\}$. Thus $w>u=u_{1}$ in $E$ and $(w-u)_{+}=0$ in $\Omega \backslash E$. Lemma 2.10 yields that there exist non-negative $u_{i} \in W^{1, \varphi(\cdot)}\left(D \cap \Omega^{\prime}\right)$ with spt $u_{i} \Subset D \cap \Omega^{\prime},\left\{u_{i} \neq 0\right\} \subset$ $\left\{(w-u)_{+} \neq 0\right\}$ and $u_{i} \rightarrow(w-u)_{+}$in $W^{1, \varphi(\cdot)}\left(D \cap \Omega^{\prime}\right)$, by assumptions (A0), (A1), (aInc) and (aDec). Since $u_{1}$ is a quasisuperminimizer in $D$ we obtain by Lemma 4.5 that

$$
\begin{aligned}
\int_{E} \varphi\left(x,\left|\nabla u_{1}\right|\right) d x & \leqslant 2^{q} L K \int_{E} \varphi\left(x,\left|\nabla\left(u_{1}+(w-u)_{+}\right)\right|\right) d x \\
& =2^{q} L K \int_{E} \varphi(x,|\nabla w|) d x \\
& =2^{q} L K \int_{E \backslash A} \varphi(x,|\nabla v|) d x+2^{q} L K \int_{E \cap A} \varphi\left(x,\left|\nabla u_{2}\right|\right) d x .
\end{aligned}
$$

If $x \in G \backslash A$, then $u_{2}(x) \geqslant v(x)>u(x)$ so we must have $x \in D$ and $u_{1}(x)<u_{2}(x)$. This means that $x \in E$. Since $A \subset G$, we obtain $G=E \cup A$. We complete the proof by using the estimates above in a suitable order:

$$
\begin{aligned}
& \int_{G} \varphi(x,|\nabla u|) d x=\int_{A \backslash E} \varphi\left(x,\left|\nabla u_{2}\right|\right) d x+\int_{E} \varphi\left(x,\left|\nabla u_{1}\right|\right) d x \\
& \quad \leqslant \int_{A \backslash E} \varphi\left(x,\left|\nabla u_{2}\right|\right) d x+K \int_{E \cap A} \varphi\left(x,\left|\nabla u_{2}\right|\right) d x+K \int_{E \backslash A} \varphi(x,|\nabla v|) d x \\
& \quad \leqslant K \int_{A} \varphi\left(x,\left|\nabla u_{2}\right|\right) d x+K \int_{E \backslash A} \varphi(x,|\nabla v|) d x \\
& \quad \leqslant 2^{q} L K^{2} \int_{A} \varphi(x,|\nabla v|) d x+K^{2} \int_{E \backslash A} \varphi(x,|\nabla v|) d x \\
& \quad=2^{q} L K^{2} \int_{G} \varphi(x,|\nabla v|) d x .
\end{aligned}
$$

This concludes the proof. 


\section{Capacity density condition for superminimizers}

In this section we continue our study of regularity properties of superminimizers. Note that we have to make two restrictions at this point compared to earlier sections: instead of quasisuperminimizers we consider superminimizers, and in place of $\Phi_{w}(\Omega)$ we have $\Phi(\Omega)$.

Lemma 5.1. Let $\varphi \in \Phi(\Omega)$ satisfy (aInc) and (aDec), and let $\alpha \in(0, p-1]$. If $u$ is a non-negative superminimizer, then

$$
\int_{B} u^{-\alpha-1} \varphi(x,|\nabla u|) d x \lesssim \int_{2 B} u^{-\alpha-1} \varphi\left(x, \frac{u}{\operatorname{diam} B}\right) d x .
$$

Proof. Let $\eta \in C_{0}^{\infty}(2 B)$ be a cut-off function: $0 \leqslant \eta \leqslant 1, \eta=1$ in $B$ and $|\nabla \eta| \lesssim \operatorname{diam}(B)^{-1}$. Let $u_{k}:=k u+1$ and $v_{k}:=\frac{1}{k \alpha} \eta^{q} u_{k}^{-\alpha}, \alpha>0$. Then

$$
\nabla v_{k}=q \eta^{q-1} \frac{u_{k}^{-\alpha}}{k \alpha} \nabla \eta-\eta^{q} u_{k}^{-\alpha-1} \nabla u
$$

and so

$$
\left|\nabla\left(u+v_{k}\right)\right| \leqslant \eta^{q} u_{k}^{-\alpha-1} \frac{q u_{k}|\nabla \eta|}{k \alpha \eta}+\left(1-\eta^{q} u_{k}^{-\alpha-1}\right)|\nabla u| .
$$

Testing with $v_{k}$ and using convexity and $\eta^{q} u_{k}^{-\alpha-1} \in[0,1]$, we find that

$$
\int_{2 B} \varphi(x, \nabla u) d x \leqslant \int_{2 B} \eta^{q} u_{k}^{-\alpha-1} \varphi\left(x, \frac{q u_{k}|\nabla \eta|}{k \alpha \eta}\right)+\left(1-\eta^{q} u_{k}^{-\alpha-1}\right) \varphi(x,|\nabla u|) d x .
$$

We then move the last term on the right to the left:

$$
\int_{2 B} \eta^{q} u_{k}^{-\alpha-1} \varphi(x,|\nabla u|) d x \lesssim \int_{2 B} \eta^{q} u_{k}^{-\alpha-1} \varphi\left(x, \frac{\left(u+\frac{1}{k}\right)|\nabla \eta|}{\eta}\right) d x .
$$

Next we multiply the equation by $k^{\alpha+1}$ and observe that $\eta^{-q} \varphi(x, c \eta)$ is almost decreasing in $\eta$. Since $\chi_{B} \leqslant \eta \leqslant \chi_{2 B}$, we obtain that

$$
\int_{B}\left(u+\frac{1}{k}\right)^{-\alpha-1} \varphi(x,|\nabla u|) d x \lesssim \int_{2 B}\left(u+\frac{1}{k}\right)^{-\alpha-1} \varphi\left(x,\left(u+\frac{1}{k}\right)|\nabla \eta|\right) d x .
$$

The left-hand side is increasing in $k$, and since $1+\alpha \leqslant p$, the right-hand side is almost decreasing in $k$. Furthermore,

$\left(u+\frac{1}{k}\right)^{-\alpha-1} \varphi\left(x,\left(u+\frac{1}{k}\right)|\nabla \eta|\right) \lesssim(u+1)^{-\alpha-1} \varphi(x,(u+1)|\nabla \eta|) \lesssim \varphi\left(x, \frac{u+1}{r}\right) \in L^{1}$, since $\varphi$ is doubling and $|\nabla \eta| \leqslant c / r$. Thus by monotone convergence (LHS) and dominated convergence (RHS) we obtain, as $k \rightarrow \infty$, that

$$
\int_{B} u^{-\alpha-1} \varphi(x,|\nabla u|) d x \lesssim \int_{2 B} u^{-\alpha-1} \varphi(x, u|\nabla \eta|) d x \lesssim \int_{2 B} u^{-\alpha-1} \varphi\left(x, \frac{u}{\operatorname{diam} B}\right) d x .
$$

This concludes the proof. 
Now we can show the fine continuity of the lower semicontinuous representative of a superminimizer.

Theorem 5.2. Let $\varphi \in \Phi\left(\mathbb{R}^{n}\right)$ satisfy (A0), (A1), (A1-n), (aInc) and (aDec). If $u$ is a non-negative superminimizer, then for every $\varepsilon>0$ and every $x_{0} \in \Omega$

$$
\frac{C_{\varphi(\cdot)}\left(B\left(x_{0}, r\right) \cap\left\{\left|u^{*}-u^{*}\left(x_{0}\right)\right|>\varepsilon\right\}, B\left(x_{0}, 2 r\right)\right)}{C_{\varphi(\cdot)}\left(B\left(x_{0}, r\right), B\left(x_{0}, 2 r\right)\right)} \rightarrow 0
$$

as $r \rightarrow 0^{+}$. Here $u^{*}$ is the lower semicontinuous representative of $u$ defined in Theorem 4.4.

Proof. We may assume that $\varepsilon \in(0,1]$. For simplicity we denote $u^{*}$ by $u$. By Theorem 4.4, we know that $u\left(x_{0}\right)=\liminf _{x \rightarrow x_{0}} u(x)$. Thus there exists $r_{0}>0$ such that $B\left(x_{0}, r_{0}\right) \cap\left\{x \in \Omega: u(x)<u\left(x_{0}\right)-\varepsilon\right\}=\emptyset$. So let us study $E:=\{x \in \Omega: u(x)>l\}$ with $l:=u\left(x_{0}\right)+\varepsilon$. We assume that $r \in\left(0, \frac{1}{4} r_{0}\right)$ and $\varepsilon \in\left(0, \frac{1}{4}\right)$ and denote $B:=B\left(x_{0}, r\right)$.

Let $\eta \in C_{0}^{\infty}(2 B)$ be such that $0 \leqslant \eta \leqslant 1, \eta=1$ in $B$ and $|\nabla \eta| \lesssim r^{-1}$. Let $m(r):=\inf _{B\left(x_{0}, r\right) \cap \Omega} \min \{u, l\}$ and $v:=\min \{u, l\}-m(4 r)$. Then $E \cap B \subset$ $\left\{\frac{2}{\varepsilon} v \eta>1\right\}$ and by Lemma $4.6 v$ is a non-negative superminimizer.

Since $u$ is lower semicontinuous and $\eta$ is continuous, the set $\left\{\frac{2}{\varepsilon} v \eta>1\right\}$ is open. Thus $2 v \eta$ is suitable test function for the capacity and we obtain

$C_{\varphi(\cdot)}(B \cap E, 2 B) \leqslant \int_{2 B} \varphi\left(x,\left|\nabla\left(\frac{2}{\varepsilon} v \eta\right)\right|\right) d x \leqslant \int_{2 B} \varphi\left(x, \frac{4}{\varepsilon} \eta|\nabla v|\right)+\varphi\left(x, \frac{4}{\varepsilon} v|\nabla \eta|\right) d x$ since $|\nabla(c v \eta)| \leqslant 2 \max \{c \eta|\nabla v|, c v|\nabla \eta|\}$. Using $\eta \leqslant 1$, doubling and $v \leqslant 2$ we find that

$$
\varphi\left(x, \frac{4}{\varepsilon} \eta|\nabla v|\right) \leqslant \varphi\left(x, \frac{4}{\varepsilon}|\nabla v|\right) \lesssim \varphi(x,|\nabla v|) \lesssim v^{-\alpha-1} \varphi(x,|\nabla v|),
$$

where the implicit constant depends on $\varepsilon$. Then it follows from Lemma 5.1 that

$$
\int_{2 B} \varphi\left(x, \frac{4}{\varepsilon} \eta|\nabla v|\right) d x \lesssim \int_{2 B} v^{-\alpha-1} \varphi(x,|\nabla v|) d x \lesssim \int_{4 B} v^{-\alpha-1} \varphi\left(x, \frac{v}{r}\right) d x .
$$

By doubling, the definition of $\eta$ and $v \leqslant 2$, we have $\varphi\left(x, \frac{4}{\varepsilon} v|\nabla \eta|\right) \lesssim \varphi\left(x, \frac{v}{r}\right) \leqslant$ $v^{-\alpha-1} \varphi\left(x, \frac{v}{r}\right)$, where the implicit constant depends on $\varepsilon$.

Since $v \leqslant 2$, it follows from (aInc) that $\varphi\left(x, \frac{v}{r}\right) \lesssim v^{p} \varphi_{4 B}^{+}\left(\frac{1}{r}\right)$. These estimates imply that

$$
C_{\varphi(\cdot)}(B \cap E, 2 B) \lesssim \int_{4 B} v^{-\alpha-1} \varphi\left(x, \frac{v}{r}\right) d x \lesssim \varphi_{4 B}^{+}\left(\frac{1}{r}\right) \int_{4 B} v^{p-\alpha-1} d x .
$$


We choose $\alpha \in(0, p-1)$ so large that the exponent of $v$ is less than or equal to the exponent $h$ in the weak Harnack inequality, Theorem 4.3. Then

$$
\begin{aligned}
C_{\varphi(\cdot)}(B \cap E, 2 B) & \leqslant \varphi_{4 B}^{+}\left(\frac{1}{r}\right) r^{n} \inf _{2 B}(v+r)^{p-\alpha-1} \\
& =\varphi_{4 B}^{+}\left(\frac{1}{r}\right) r^{n}(m(2 r)-m(4 r)+r)^{p-\alpha-1} .
\end{aligned}
$$

By Lemma 2.8, $C_{\varphi(\cdot)}(B, 2 B) \gtrsim \varphi_{2 B}^{-}\left(\frac{1}{r}\right) r^{n}$ and by $(\mathrm{A} 1-n), \varphi_{4 B}^{+}\left(\frac{1}{r}\right) \lesssim \varphi_{2 B}^{-}\left(\frac{1}{r}\right)$. Since $m(r)$ is bounded and decreasing, it has a limit at 0 . Thus $m(2 r)-$ $m(4 r)+r \rightarrow 0$ as $r \rightarrow 0$, and so the result follows. This concludes the proof.

Remark 5.3. In the previous theorem, if $u$ is $a$-Hölder continuous, then $m(2 r)-$ $m(4 r)+r \lesssim r^{a}$ and we get a quantitative bound for the decay with a constant depending on $\varepsilon$.

\section{Regular boundary points}

Definition 6.1. Let $\Omega \subset \mathbb{R}^{n}$ be bounded and $f \in W^{1, \varphi(\cdot)}(\Omega)$. We say that $u \in W^{1, \varphi(\cdot)}(\Omega)$ is a minimizer with boundary values $f \in W^{1, \varphi(\cdot)}(\Omega)$ if $u-f \in$ $W_{0}^{1, \varphi(\cdot)}(\Omega)$ and

$$
\int_{\Omega} \varphi(x,|\nabla u|) d x \leqslant \int_{\Omega} \varphi(x,|\nabla(u+v)|) d x
$$

for all $v \in W_{0}^{1, \varphi(\cdot)}(\Omega)$.

We denote by $H(f)$ the minimizer with boundary values $f \in W^{1, \varphi(\cdot)}(\Omega)$. If $f: \partial \Omega \rightarrow \mathbb{R}$ is Lipschitz on the boundary of $\Omega$, then it can be, by McShane extension, extend to $\mathbb{R}^{n}$ as a bounded Lipschitz function. The extension of $f$ can be used in the above definition as weak boundary value, $u-f \in W_{0}^{1, \varphi(\cdot)}(\Omega)$. For $g \in C(\partial \Omega)$ we define

$$
H_{g}(x):=\sup _{f \leqslant g, f \text { is Lipschitz }} H(f)(x) .
$$

This definition is based on the fact continuous function can be approximated by Lipschitz functions.

We have previously shown existence of minimizers with given Dirichlet boundary values $f \in W^{1, \varphi(\cdot)}(\Omega)$ in Theorem 7.3 of [17]. However, if $f \in C(\partial \Omega)$, the same conclusion can be reached under fewer assumptions on $\varphi$.

Theorem 6.2. Let $\Omega \subset \mathbb{R}^{n}$ be bounded. Let $\varphi \in \Phi(\Omega)$ satisfy (aInc) and (aDec). Then for every $f \in W^{1, \varphi(\cdot)}(\Omega) \cap L^{\infty}(\Omega)$, there exists a minimizer $H(f)$.

If $\varphi$ is strictly convex and satisfies (AO), the minimizer is unique, and if (A1-n) holds, then it is continuous. 
Proof. Let $M>0$ be such that $|f| \leqslant M$ a.e. If $u_{M}$ is a cut-off of $u$ at levels $-M$ and $M$, then

$$
\int_{\Omega} \varphi\left(x,\left|\nabla u_{M}\right|\right) d x \leqslant \int_{\Omega} \varphi(x,|\nabla u|) d x
$$

Thus we conclude that the possible minimizer satisfies $|u| \leqslant M$.

Let $u_{i} \in W^{1, \varphi(\cdot)}(\Omega)$ be a sequence of functions with $u_{i}-f \in W_{0}^{1, \varphi(\cdot)}(\Omega)$ and

$$
\inf _{u} \int_{\Omega} \varphi(x,|\nabla u|) d x=\lim _{i \rightarrow \infty} \int_{\Omega} \varphi\left(x,\left|\nabla u_{i}\right|\right) d x .
$$

We assume without loss of generality that $\left|u_{i}\right| \leqslant M$. Then $\varrho_{1, \varphi(\cdot)}\left(u_{i}\right)$ is uniformly bounded, and so $\left(u_{i}\right)$ is a bounded sequence in $W^{1, \varphi(\cdot)}(\Omega)$ [10, Corollary 2.1.15]. By [16], $W^{1, \varphi(\cdot)}(\Omega)$ is a reflexive Banach space, and so $\left(u_{i}\right)$ has a weakly convergent subsequence. Since $\varrho_{\varphi(\cdot)}$ is weakly lower semicontinuous [10, Theorem 2.2.8], the weak limit $u$ satisfies

$$
\int_{\Omega} \varphi(x,|\nabla u|) d x \leqslant \lim _{i \rightarrow \infty} \int_{\Omega} \varphi\left(x,\left|\nabla u_{i}\right|\right) d x=\inf _{u} \int_{\Omega} \varphi(x,|\nabla u|) d x
$$

and hence $u$ is the minimizer.

When $\varphi \in \Phi$ is strictly convex and satisfies (A0), the possible minimizer is unique by Theorem 7.5 of [17]. If $\varphi \in \Phi(\Omega)$ satisfies (A0), (A1-n), (aInc) and (aDec), a locally bounded minimizer is locally Hölder continuous by [18, Corollary 1.5] (note that assumption (A1) is then not needed, cf. [18, Theorem 5.7].) This concludes the proof.

Definition 6.3. Let $\Omega \subset \mathbb{R}^{n}$. We say that $x \in \partial \Omega$ is regular if

$$
\lim _{y \rightarrow x, y \in \Omega} H_{f}(y)=f(x)
$$

for all $f \in C(\partial \Omega)$. A boundary point is irregular if it is not regular.

This means that the minimizer attains the boundary values not only in a Sobolev sense but point-wise. The next lemma gives a comparison principle for minimizers.

Lemma 6.4. Let $\Omega \subset \mathbb{R}^{n}$ be bounded. Let $\varphi \in \Phi(\Omega)$ be strictly convex and satisfy (A0), (A1), (A1-n), (aInc) and (aDec). Let $f, g \in W^{1, \varphi(\cdot)}(\Omega) \cap L^{\infty}(\Omega)$. If $f \leqslant g$ almost everywhere in $\Omega$, then $H(f) \leqslant H(g)$ everywhere in $\Omega$.

Proof. It follows from Theorem 6.2 that $H(f)$ and $H(g)$ exist and are unique and continuous. 
Let $u:=\min \{H(f), H(g)\}$ and $h:=H(f)-f-(H(g)-g) \in W_{0}^{1, \varphi(\cdot)}(\Omega)$. Then

$$
f+H(g)-g+\min \{g-f, h\}=\min \{H(g), h+f+H(g)-g\}=u
$$

so that $u-f=H(g)-g+\min \{g-f, h\}$. Then $u-f \in W_{0}^{1, \varphi(\cdot)}(\Omega)$, since $H(g)-g \in W_{0}^{1, \varphi(\cdot)}(\Omega)$ and by Lemma $2.11 \min \{g-f, h\} \in W_{0}^{1, \varphi(\cdot)}(\Omega)$.

Similarly we obtain for $v:=\max \{H(f), H(g)\}$ that $v-g \in W_{0}^{1, \varphi(\cdot)}(\Omega)$. Let $A:=\{H(f) \geqslant H(g)\}$. Since $H(g)$ is a minimizer

$$
\begin{aligned}
\int_{\Omega} \varphi(x,|\nabla H(g)|) d x & \leqslant \int_{\Omega} \varphi(x,|\nabla v|) d x \\
& =\int_{A} \varphi(x,|\nabla H(f)|) d x+\int_{\Omega \backslash A} \varphi(x,|\nabla H(g)|) d x
\end{aligned}
$$

and so

$$
\int_{A} \varphi(x,|\nabla H(g)|) d x \leqslant \int_{A} \varphi(x,|\nabla H(f)|) d x .
$$

Thus we obtain that

$$
\begin{aligned}
\int_{\Omega} \varphi(x,|\nabla u|) d x & =\int_{A} \varphi(x,|\nabla H(g)|) d x+\int_{\Omega \backslash A} \varphi(x,|\nabla H(f)|) d x \\
& \leqslant \int_{\Omega} \varphi(x,|\nabla H(f)|) d x
\end{aligned}
$$

and hence $u$ is a minimizer with Sobolev boundary values $f$. Since by Theorem 6.2 the minimizer is unique, we obtain that $H(f)=u=\min \{H(f), H(g)\}$ almost everywhere. This yields that $H(f) \leqslant H(g)$ almost everywhere, and since both are continuous this holds everywhere. This concludes the proof.

The previous lemma yields the following fact: If $f, g \in C(\partial \Omega)$ and $f \leqslant g$, then $H_{f} \leqslant H_{g}$. The proof follows the proof of Lemma 7.6 in [4]. The next proof follows the outlines given in Lemma 2.132, p. 141, of [23]

Proposition 6.5. Let $\Omega \subset \mathbb{R}^{n}$ be bounded. Let $\varphi \in \Phi(\Omega)$ be strictly convex and satisfy (A0), (A1), (A1-n), (aInc) and (aDec). If $\lim _{y \rightarrow x, y \in \Omega} H(f)(y)=f(x)$ holds for every $f \in C_{0}^{\infty}\left(\mathbb{R}^{n}\right)$, then $x$ is regular.

Proof. Let $g \in C(\partial \Omega)$. We extend $g$, via Tietze's theorem or Urysohn's lemma, to a function in $C_{0}\left(\mathbb{R}^{n}\right)$. This extension is denoted again by $g$. For $\varepsilon>0$, let $f \in C_{0}^{\infty}\left(\mathbb{R}^{n}\right)$ be such that $|f-g|<\varepsilon$ in $\mathbb{R}^{n}$, see for example Theorem 4.1 in [11]. By Lemma 6.4,

$$
H(f)-\varepsilon=H(f-\varepsilon) \leqslant H_{g} \leqslant H(f+\varepsilon)=H(f)+\varepsilon .
$$


Then, for $z \in \partial \Omega$,

$$
g(z)-2 \varepsilon \leqslant f(z)-\varepsilon \leqslant \liminf _{x \rightarrow z, x \in \Omega} H_{g}(x) \leqslant \limsup _{x \rightarrow z, x \in \Omega} H_{g}(x) \leqslant f(z)+\varepsilon \leqslant g(z)+2 \varepsilon
$$

and the claim follows as $\varepsilon \rightarrow 0^{+}$. This concludes the proof.

Proof of Theorem 1.1. By Proposition 6.5, we may assume that $f \in C_{0}^{\infty}\left(\mathbb{R}^{n}\right)$ in the definition of regular boundary points. Let $H(f)$ be the continuous minimizer with boundary values $f$ given by Theorem 6.2 . Choose $k<f\left(x_{0}\right)$. Then there exists $r>0$ such that $f>k$ in $\bar{B}\left(x_{0}, r\right) \backslash \Omega$. Let

$$
u:= \begin{cases}\min \{H(f), k\} & \text { in } B\left(x_{0}, r\right) \cap \Omega \\ k & \text { in } B\left(x_{0}, r\right) \backslash \Omega .\end{cases}
$$

Next we show that $u$ is a Sobolev function. Since $H(f)-f \in W_{0}^{1, \varphi(\cdot)}(\Omega)$, the function

$$
g:= \begin{cases}H(f)-f & \text { in } \Omega \\ 0 & \text { in } \mathbb{R}^{n} \backslash \Omega\end{cases}
$$

belongs to $W^{1, \varphi(\cdot)}\left(\mathbb{R}^{n}\right)$. Hence also $g+f \in W^{1, \varphi(\cdot)}\left(\mathbb{R}^{n}\right)$ so that $\min \{k, g+f\} \in$ $W^{1, \varphi(\cdot)}\left(\mathbb{R}^{n}\right)$. This shows that $u \in W^{1, \varphi(\cdot)}\left(B\left(x_{0}, r\right)\right)$, since $u=\min \{k, g+f\}$ in $B\left(x_{0}, r\right)$.

Since $u$ is a Sobolev function, the pasting lemma 4.6 yields that $u$ is a superminimizer in $B\left(x_{0}, r\right)$. By Theorem $4.4, u$ has a representative $u^{*}$ which is lower semicontinuous. Suppose that $u^{*}\left(x_{0}\right) \neq k$. Choose $\varepsilon:=\frac{1}{2}\left|k-u^{*}\left(x_{0}\right)\right|$ in Theorem 5.2 and note that $B\left(x_{0}, r\right) \backslash \Omega \subset\left\{\left|u^{*}-u^{*}\left(x_{0}\right)\right|>\varepsilon\right\}$ since $u^{*}=k$ in $B\left(x_{0}, r\right) \backslash \Omega$. By assumption, the boundary is such that $C_{\varphi}\left(B\left(x_{0}, r\right) \backslash\right.$ $\left.\Omega, B\left(x_{0}, 2 r\right)\right) \geqslant c C_{\varphi}\left(B\left(x_{0}, r\right), B\left(x_{0}, 2 r\right)\right)$ for all sufficiently small $r$. This contradicts the conclusion of Theorem 5.2. Hence $u^{*}\left(x_{0}\right)=k$.

Since $u^{*}$ is lower semicontinuous we obtain that

$$
\liminf _{x \rightarrow x_{0}, x \in \Omega} H(f)(x) \geqslant \liminf _{x \rightarrow x_{0}, x \in \Omega} u^{*}(x) \geqslant u^{*}\left(x_{0}\right)=k .
$$

Since this holds for all $k<f\left(x_{0}\right)$, we obtain that $\liminf _{x \rightarrow x_{0}, x \in \Omega} H(f)(x) \geqslant$ $f\left(x_{0}\right)$.

The previous result for $-f$ yields that $\liminf _{x \rightarrow x_{0}, x \in \Omega} H(-f)(x) \geqslant-f\left(x_{0}\right)$. Since $H(-f)=-H(f)$ we obtain that $\lim _{\sup _{x \rightarrow x_{0}, x \in \Omega}} H(f)(x) \leqslant f\left(x_{0}\right)$, so $x_{0}$ is regular. This concludes the proof.

\section{Acknowledgement}

We thank Arttu Karppinen and the referee for some useful comments. 


\section{References}

[1] M. Avci and A. Pankov: Multivalued elliptic operators with nonstandard growth, Adv. Nonlinear Anal. 7 (2018), no. 1, 35-48.

[2] P. Baroni, M. Colombo and G. Mingione: Harnack inequalities for double phase functionals, Nonlinear Anal. 121 (2015), 206-222.

[3] P. Baroni, M. Colombo and G. Mingione: Non-autonomous functionals, borderline cases and related function classes, St Petersburg Math. J. 27 (2016), $347-379$.

[4] A. Björn and J. Björn: Nonlinear potential theory on metric spaces, EMS Tracts in Mathematics, 17, European Mathematical Society (EMS), Zürich, 2011.

[5] F. Colasuonno and M. Squassina: Eigenvalues for double phase variational integrals, Ann. Mat. Pura Appl. (4) 195 (2016), no. 6, 1917-1959.

[6] M. Colombo and G. Mingione: Regularity for double phase variational problems, Arch. Ration. Mech. Anal. 215 (2015), no. 2, 443-496.

[7] M. Colombo and G. Mingione: Bounded minimisers of double phase variational integrals, Arch. Ration. Mech. Anal. 218 (2015), no. 1, 219-273.

[8] M. Colombo and G. Mingione: Calderón-Zygmund estimates and nonuniformly elliptic operators, J. Funct. Anal. 270 (2016), 1416-1478.

[9] D. Cruz-Uribe and P. Hästö: Extrapolation and interpolation in generalized Orlicz spaces, Trans. Amer. Math. Soc. 370 (2018), no. 6, 4323-4349.

[10] L. Diening, P. Harjulehto, P. Hästö and M. Růžička: Lebesgue and Sobolev spaces with variable exponents, Lecture Notes in Mathematics, 2017. Springer, Heidelberg, 2011.

[11] L. C. Evans and R. F. Gariepy: Measure theory and fine properties of functions. Revised edition. Textbooks in Mathematics. CRC Press, Boca Raton, FL, 2015.

[12] F. Giannetti and A. Passarelli di Napoli: Regularity results for a new class of functionals with non-standard growth conditions, J. Differential Equations 254 (2013), 1280-1305.

[13] E. Giusti: Direct Methods in the Calculus of Variations, World Scientific, Singapore, 2003.

[14] P. Gwiazda, P. Wittbold, A. Wróblewska and A. Zimmermann: Renormalized solutions to nonlinear parabolic problems in generalized Musielak-Orlicz spaces, Nonlinear Anal. 129 (2015), 1-36.

[15] P. Harjulehto and P. Hästö: Riesz potential in generalized Orlicz Spaces, Forum Math. 29 (2017), no. 1, 229-244.

[16] P. Harjulehto and P. Hästö: Uniform convexity and associate spaces, Czechosolvak Math. J., to appear, DOI:10.21136/CMJ.2018.0054-17.

[17] P. Harjulehto, P. Hästö and R. Klén: Generalized Orlicz spaces and related PDE, Nonlinear Anal. 143 (2016), 155-173. 
[18] P. Harjulehto, P. Hästö and O. Toivanen: Hölder regularity of quasiminimizers under generalized growth conditions, Calc. Var. Partial Differential Equations 56 (2017), article 22.

[19] P. Harjulehto, T. Kuusi, T. Lukkari, N. Marola, M. Parviainen: Harnack's inequality for quasiminimizers with non-standard growth conditions, J. Math. Anal. Appl. 344 (2008), no. 1, 504-520.

[20] P. Hästö: The maximal operator on generalized Orlicz spaces, J. Funct. Anal 269 (2015), no. 12, 4038-404; 271 (2016), no. 1, 240-243.

[21] V. Latvala, and O. Toivanen: Weak Harnack estimates for quasiminimizers with non-standard growth and general structure, Potential Anal. 47 (2017), no. 1, 21-36.

[22] F.-Y. Maeda, Y. Mizuta, T. Ohno and T. Shimomura: Boundedness of maximal operators and Sobolev's inequality on Musielak-Orlicz-Morrey spaces, Bull. Sci. Math. 137 (2013), 76-96.

[23] J. Malý and W. P. Ziemer: Fine regularity of solutions of elliptic partial differential equations, Mathematical Surveys and Monographs, 51. American Mathematical Society, Providence, RI, 1997.

[24] J. Musielak: Orlicz spaces and modular spaces, Lecture Notes in Mathematics, 1034. Springer, Berlin, 1983.

[25] T. Ohno and T. Shimomura: Musielak-Orlicz-Sobolev spaces with zero boundary values on metric measure spaces, Czechoslovak Math. J. 66(141) (2016), no. $2,371-394$.

[26] J. Ok: Gradient estimates for elliptic equations with $L^{p(\cdot)} \log L$ growth, Calc. Var. Partial Differential Equations 55 (2016), no. 2, 1-30.

[27] L. Pick and M. Růžička: An example of a space $L^{p(x)}$ on which the HardyLittlewood maximal operator is not bounded, Expo. Math. 19 (2001), no. 4, 369-371.

Received April 18, 2017; revised October 27, 2018 